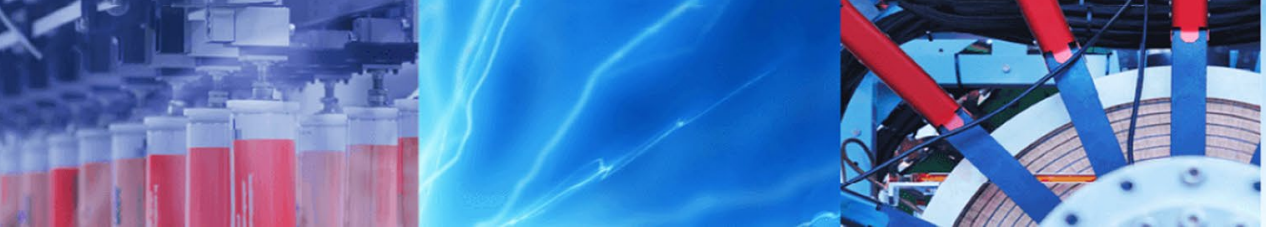

Research Article

\title{
Keller-box analysis of inclination flow of magnetized Williamson nanofluid
}

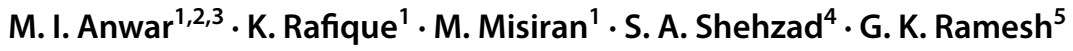

Received: 21 November 2019 / Accepted: 9 January 2020 / Published online: 10 February 2020

(c) Springer Nature Switzerland AG 2020

\begin{abstract}
The main purpose of the current investigation is to report the numerical solution of the thermal radiations and MHD effect on the laminar flow of an incompressible Williamson nanofluid. Further, the effect of Brownian motion and thermophoresis on the flow field are considered. Compatible similarities are implemented on the flow equations to obtain the nonlinear ordinary differential equations. The numerical solution of the governing differential equations is obtained via the Keller box scheme. Findings exhibit that the energy and mass transport moderates with the growth of inclination factor along with Brownian motion effect. The temperature profile increases with the radiations factor attains a peak, which is useful for the industrial procedures. The velocity profile increases for the higher magnitudes of buoyancy factor.
\end{abstract}

Keywords Williamson nanofluid · Thermal radiations · Inclination · MHD · Inclined surface

\section{Introduction}

At this time, the analysis of heat exchange via nanofluids become a hot area of research for the latest investigators. It is because of the base liquids such as lubricants, ethylene glycol, oil, water, and kerosene, etc. have become less promising in a couple of instances of uses. Hence, a novel type of liquids is required to acquire the thermal efficiencies for energy exchanger in the forthcoming ventures. Choi [1] originated a new thoughtful plan to determine the problem by including the dilute suspensions of nanomaterials into the base liquids, which termed as nanofluids. Nanofluids are a mixture of metals, carbides, nonmetals, oxides, carbides, and have measurements of 1-100 nm. Due to the modest size of nanomaterials, nanoliquids have solid suspension strength and capacity to transport deprived of clogging the flow structure. Since the nanomaterials have better thermal efficiency than the base liquid, nanofluids viewed as better coolants especially in atomic reactors, domestic freezers, malignant growth treatment, electronic gadgets, oils, and furthermore thinfilm sun oriented vitality collectors. The nanoparticles play a dynamic role in nanotechnology as well in the medical field for instance in hyperthermia cancer cure and in medical procedures. Further, in hybrid power devices and microelectronics [2]. Due to the abovementioned applications, numerous investigators began to utilize nanofluid as an elective method to upgrade the energy exchange adequacy. For example, Buongiorno [3] proposed the nonhomogeneous equilibrium model, which contained the following slip components; inertia, diffusiophoresis, thermophoresis, Brownian motion effect, gravity Magnus influence, and liquid drainage. Thermophoretic and Brownian motion effects are more prominent in the enhancement of thermal conductivity of the base liquids in this model. Tiwari and Das [4] offered a homogeneous model by incorporating the nanoparticle fractions effects. There are different models in the literature for instance Buongiorno

$\triangle$ S. A. Shehzad, ali_qau70@yahoo.com | 'School of Quantitative Sciences, Universiti Utara Malaysia, 06010 Sintok, Kedah, Malaysia. ${ }^{2}$ Department of Mathematics, Faculty of Science, University of Sargodha, Sargodha, Pakistan. ${ }^{3}$ Higher Education Department (HED) Punjab, Lahore, Pakistan. ${ }^{4}$ Department of Mathematics, COMSATS University Islamabad, Sahiwal 57000, Pakistan. ${ }^{5}$ Department of Mathematics, K.L.E Society's J.T. College, Gadag, Karnataka 582101, India. 
model, Tewari and Das model etc. but in current article we consider the Buongiorno model the reason behind its Brownnian motion thermophoretic impacts trigger the energy transport phenomeon. Some related literature on the homogeneous and non-homogeneous models available in the references [5-15].

Several specialists revealed the MHD importance in several energy based flows generated by stretching sheet. It has received much consideration due to its significant applications in the engineering and practical field such as MHD power producers, hyperthermia cancer cure, brain tumor treatment etc. The physical effects of magnetized material offer influential circustances in heat transport fluid flow problems. The Lenz's law reports that electric current produced by movement of conductor under magnetic field strength that comprises the magnetic field. The movement of fluid changes when conducting liquid moves under the magnetic field, and the magnetized nanoparticles interact through Lorentz forces. For brief information on magnetized material properties, one can see the investigator reports on MHD flows in the references [11, 16-21].

Heat exchange because of thermal radiations considered as an active part of investigation reason is its wide variety of uses in atomic plants, nanotechnology, missiles, and in satellites. Moreover, it is noteworthy thermal radiation not appropriate in manufacturing of the thermal devices with a significant variation in heat. Pal and Roy [22] studied the thermal radiation effect on the flow of nanofluid on a sheet. Latest, Ghadikolaei et al. [23] probed
For a detailed knowledge about the Williamson nanofluid flow with different impacts see [27-30].

Because of the literature mentioned above the novelty of the current study is to scrutinize the inclination effect on the Williamson nanofluid flow towards an inclined surface by incorporating the impact of the thermal radiations. The Brownian motion and thermophoretic force took into account. By the authors best knowledge, there is no study available on the problem under consideration. The boundary layer equations converted into the ordinary differential equation by employing the compatible transformations. The Keller box scheme is utilized for the numerical simulation of the study under concern [31].

\section{Mathematical formulation}

Here, we assumed the incompressible flow of Williamson type nanofluid over an inclined surface. The thermal radiations along with magnetic effect are carried out in this work. The inclination between the vertical and fluid flow direction is $\Omega$. Non-Newtonian Williamson parameter and buoyancy factors effects the flow filed. The wall temperature and nanoparticle concentration are signifies by $T_{w}, C_{w}$ respectively. Moreover, the free stream temperature and conecentration are considered as $T_{\infty}, C_{\infty}$ (see Fig. 1).

The flow field is labelled by the equations as [27, 30]:

$\frac{\partial v}{\partial y}+\frac{\partial u}{\partial x}=0$

$v \frac{\partial v}{\partial y}+u \frac{\partial u}{\partial x}=v \frac{\partial^{2} u}{\partial y^{2}}+\sqrt{2} v \Gamma \frac{\partial u}{\partial y} \frac{\partial^{2} u}{\partial y^{2}}+g \cos \Omega\left[\beta_{c}\left(C-C_{\infty}\right)+\beta_{t}\left(T-T_{\infty}\right)\right]-\frac{\sigma B_{0}^{2}}{\rho} u$,

the Casson nanofluid flow on the permeable slanted sheet numerically. Saidulu [24] argued the radiation influences on the stream of the nanofluid on slanted surface.

In modern centuries, scholars give more intention to the boundary layer flow of non-Newtonian fluids. Moreover, their rising requirement in industries and engineering increasing the interest of several researchers to try to know the features of these so-called rheological liquids in addition to their complex behavior so far fascinating at the same time. The pseudoplastic fluid is one of the attention-grabbing fluid among the non-Newtonian fluids. The pseudoplastic fluid flow has gained much importance because of its application in industrial and engineering procedures. The Williamson liquid model was presented to reveal the performance of this kind of liquids. The pseudoplastic flow constituents discussed by Williamson [25]. Vijayalaxmi and Shankar [26] investigated the flow of Williamson nanofluid through an exponential inclined sheet.

$$
v \frac{\partial T}{\partial y}+u \frac{\partial T}{\partial x}=\alpha \frac{\partial^{2} T}{\partial y^{2}}-\frac{1}{(\rho C)_{f}} \frac{\partial q_{r}}{\partial y}+\tau\left[\frac{\partial C}{\partial y} \frac{\partial T}{\partial y} D_{B}+\left(\frac{\partial T}{\partial y}\right)^{2} \frac{D_{T}}{T_{\infty}}\right]
$$

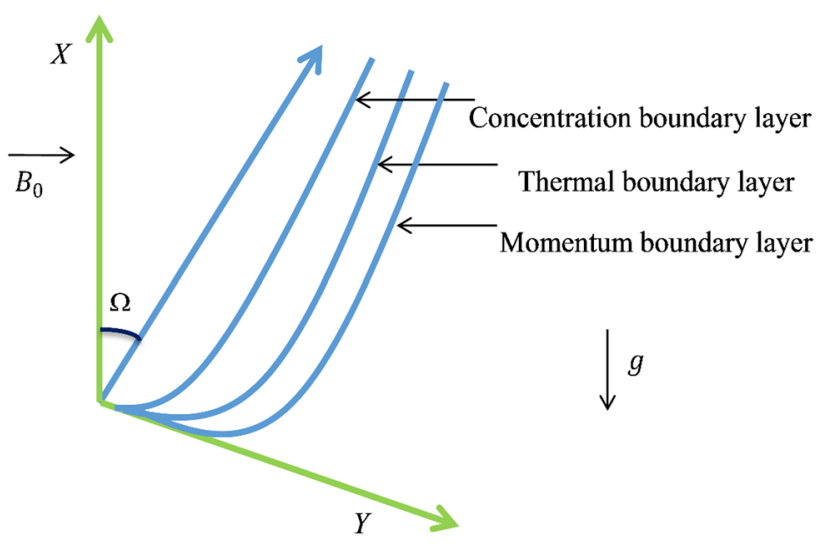

Fig. 1 Physical structure with coordinates 
$v \frac{\partial C}{\partial y}+u \frac{\partial C}{\partial x}=\frac{D_{T}}{T_{\infty}} \frac{\partial^{2} T}{\partial y^{2}}+D_{B} \frac{\partial^{2} C}{\partial y^{2}}$.

Here, the Rosseland estimation (for radiation flux) characterized as

$q_{r}=-\frac{\partial T^{4}}{\partial y} \frac{4 \sigma^{*}}{3 k^{*}}$

where $\sigma^{*}$ denotes the Stefan-Boltzmann factor and $k^{*}$ represents the mean absorption constant. Whereas

$T^{4} \cong-3 T_{\infty}^{4}+4 T_{\infty}^{3} T$.

By the virtue of Eqs. (5) and (6), the expression (3) converted into:

$v \frac{\partial T}{\partial y}+u \frac{\partial T}{\partial x}=\left(\alpha+\frac{16 \sigma^{*} T_{\infty}^{3}}{3 k^{*}(\rho c)_{f}}\right) \frac{\partial^{2} T}{\partial y^{2}}+\tau\left[\frac{\partial C}{\partial y} \frac{\partial T}{\partial y} D_{B}+\left(\frac{\partial T}{\partial y}\right)^{2} \frac{D_{T}}{T_{\infty}}\right]$.

The conditions at boundary are [31]:

$v=0, u=u_{w}(x)=a x, C=C_{w}, T=T_{w}, \quad$ at $\quad y=0$,

$v \rightarrow 0, u \rightarrow u_{\infty}(x)=0, \quad C \rightarrow C_{\infty}, \quad T \rightarrow T_{\infty}, \quad$ as $\quad y \rightarrow \infty$.

Here, the stream function $\psi=\psi(y, x)$ and similarity transformations are demarcated as

$u=\frac{\partial \psi}{\partial y}, \quad v=-\frac{\partial \psi}{\partial x}$.

$u=\operatorname{axf}(\eta), v=-\sqrt{a v} f(\eta), \eta=y \sqrt{\frac{a}{v}}$,

$\theta(\eta)=\frac{T-T_{\infty}}{T_{w}-T_{\infty}}, \phi(\eta)=\frac{C-C_{\infty}}{C_{w}-C_{\infty}}$.

This transformation convert the above expressions into following forms:

$f^{\prime \prime \prime}+f f^{\prime \prime}+\gamma_{1} f^{\prime} f^{\prime \prime \prime}-f^{\prime 2}+\cos \Omega\left(G r_{x} \theta+G c_{x} \phi\right)-M f^{\prime}=0$,

$(\underset{N}{\operatorname{Pr}}) \theta^{\prime \prime}+N b \phi^{\prime} \theta^{\prime}+f \theta^{\prime}+N t \theta^{\prime 2}=0$

$\phi^{\prime \prime}+$ Lef $\phi^{\prime}+\frac{N t}{N b} \theta^{\prime \prime}=0$.

Here

$M=\frac{\sigma B_{0}^{2}}{a \rho}, L e=\frac{v}{D_{B}}, \operatorname{Pr}=\frac{v}{\alpha}, N b=\frac{\tau D_{B}\left(C_{w}-C_{\infty}\right)}{v}, N t=\frac{\tau D_{T}\left(T_{w}-T_{\infty}\right)}{v T_{\infty}}, G r_{x}=\frac{g \beta_{t}\left(T_{w}-T_{\infty}\right) x^{-1}}{a^{2}}$,

$\operatorname{Re}_{x}=\frac{u_{w}(x) x}{v}, G c_{x}=\frac{\left(C_{w}-C_{\infty}\right) x^{-1} g \beta_{c}}{a^{2}}, N t_{b}=\frac{N t}{N b}, \gamma_{1}=\Gamma x \sqrt{\frac{2 a^{3}}{v}}, \underset{N}{\operatorname{Pr}}=\frac{1}{\operatorname{Pr}}\left(1+\frac{4}{3} N\right)$.
In above expression, $G r_{x}$ is the local Grashof number and $G c_{x}$ is the modified local Grashof number. Here, it is worth menstioning that for similarity solution $G r_{x}$ and $G c_{x}$ should be free of $x$. This condition is achieved if the thermal expansion coefficient $\beta_{t}$ and concentration expansion coefficient $\beta_{c}$ are proportional to $x^{1}$. Hence, we assume that (see references [27-30]).

$\beta_{t}=n x^{1}, \beta_{c}=n_{1} x^{1}$.

where $n$ and $n_{1}$ are constants. Substituting Eq. (15) into the parameters $G r_{x}$ and $G c_{x}$, we get

$G r=\frac{g n\left(T_{w}-T_{\infty}\right)}{a^{2}}, \quad G c=\frac{g n_{1}\left(C_{w}-C_{\infty}\right)}{a^{2}}$.

The equivalent boundary settings are changed to

$f(\eta)=0, f^{\prime}(\eta)=1, \theta(\eta)=1, \phi(\eta)=1, \quad$ at $\quad \eta=0$,

$f^{\prime}(\eta) \rightarrow 0, \theta(\eta) \rightarrow 0, \phi(\eta) \rightarrow 0, \quad$ at $\eta \rightarrow \infty$.

The prominent physical of our concern are

$C_{f}=\frac{\tau_{w}}{\rho u_{w}^{2}}, N u=\frac{x q_{w}}{k\left(T_{w}-T_{\infty}\right)}, S h=\frac{x q_{m}}{D_{B\left(C_{w}-C_{\infty}\right)}}$

where $C_{f}$ mean skin-friction, Nu mean Nusselt number and $S h$ is the Sherwood number.

Whereas, $\quad q_{m}=-D_{B} \frac{\partial C}{\partial y}, \quad q_{w}=-\left(k+\frac{4 \sigma^{*} T_{\infty}^{3}}{3 k^{*}}\right) \frac{\partial T}{\partial y}$, $\tau_{w}=\mu\left[\frac{\partial u}{\partial y}+\frac{\Gamma}{2}\left(\frac{\partial u}{\partial y}\right)^{2}\right]$ at $y=0$

The related expressions of $-\theta^{\prime}(0),-\phi^{\prime}(0)$ and $C_{f x}$ are defined as

$-\theta^{\prime}(0)=\frac{N u_{x}}{\left(1+\frac{4}{3} N\right){\sqrt{R e_{x}}}^{\prime}}-\varphi^{\prime}(0)=\frac{S h_{x}}{\sqrt{\operatorname{Re}_{x}}}$,

$C_{f x} \sqrt{\operatorname{Re}_{x}}=f^{\prime \prime}(0)+\frac{\gamma_{1}}{2} f^{\prime \prime}(0)$.

where $\operatorname{Re}_{x}=u_{w}(x) x / v$, known as the local Reynolds number.

\section{Results and Discussion}

This part presents the numerical consequences of thermal radiations effect $N$, Brownian movement factor $N b$, Grashof number $\mathrm{Gr}$, thermophoresis $N t$, magnetic field factor $M$, modified Grashof number Gc, inclination factor $\Omega$, Prandtl 
number i.e. Pr, Williamson factor $\gamma_{1}$, and Lewis number Le through graphs and tables. Table 1 is prepared in the deficiency of $G r, G c, N, M, \gamma_{1}$ and taking factor $\operatorname{Pr}=L e=10$ with $\Omega=90 \circ$. The numerical outcomes are established brilliant settlement with already published literature [32]. The effects on $-\theta^{\prime}(0),-\phi^{\prime}(0)$ and $C_{f x}(0)$ for distinct numeric values of constraints are addressed in Table 2 . Here, we found that $-\theta^{\prime}(0)$ diminishes by increasing $N b, N t, L e, M, \gamma_{1}$, and $\Omega$ whereas oppsite effect seen for $\operatorname{Pr}, G r, G c$, and $N$. Moreover, $-\phi^{\prime}(0)$ rises by improving $N b, N t, L e, G r, G c$, and $\gamma_{1}$ while its declines against the cumulative magnitudes of $N, M, \operatorname{Pr}$, and $\Omega$. Moreover, $C_{f x}(0)$ increases by increasing the magnitudes of $N b, L e, M, \gamma_{1}$, and $\Omega$. In addition, $C_{f x}(0)$ declines with the enlargement of $N t, \operatorname{Pr}, N, G r$, and $G c$.

\subsection{Velocity profile}

For velocity profile against different parameters presented see Figs. 2, 3, 4, 5 and 6 . The behavior of the velocity profile corresponds to magnetic factor is reported in Fig. 2. It is observed that $f^{\prime}(\eta)$ diminishes as we strengthen the magnetic field because it produces resistive force (Lorentz force) which slow down the fluid motion in return the velocity field reduces. Moreover, $f^{\prime}(\eta)$ enhances on the growth of Gr shown in Fig. 3. Physically, on improving

Table 1 Contrast of $-\theta^{\prime}(0)$ and $-\phi^{\prime}(0)$ against $M, N, G r, G c, \gamma_{1}=0$ with $L e, \operatorname{Pr}=10$ and $\Omega=90$ 。

\begin{tabular}{lllllll}
\hline$N b$ & $N t$ & \multicolumn{2}{c}{ Khan and pop [32] } & & \multicolumn{2}{c}{ Recent results } \\
\cline { 3 - 4 } \cline { 6 - 7 } & & $-\theta^{\prime}(0)$ & $-\phi^{\prime}(0)$ & & $-\theta^{\prime}(0)$ & $-\phi^{\prime}(0)$ \\
\hline 0.10 & 0.10 & 0.9524 & 2.1294 & & 0.95244 & 2.12945 \\
0.20 & 0.20 & 0.3654 & 2.5152 & & 0.36547 & 2.51520 \\
0.30 & 0.30 & 0.1355 & 2.6088 & & 0.13552 & 2.60888 \\
0.40 & 0.40 & 0.0495 & 2.6038 & & 0.04951 & 2.60384 \\
0.50 & 0.50 & 0.0179 & 2.5731 & & 0.01790 & 2.57312 \\
\hline
\end{tabular}

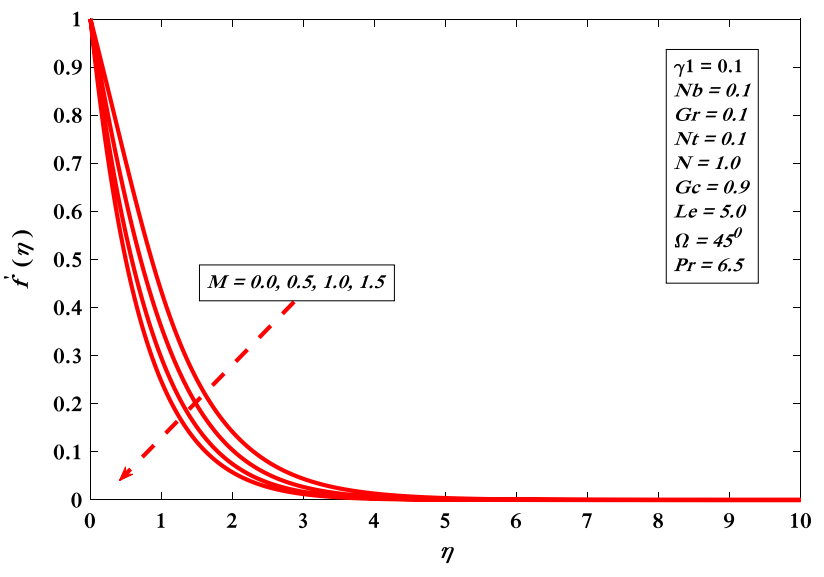

Fig. 2 Velocity $f^{\prime}(\eta)$ verses distinct values of $M$

the buoyancy impacts the viscous force turns to reduce which increases the fluid motion. Fig 4 indicates the relationship between the solutal buoyancy forces and velocity profile. Physically, the parameter Gc show its impact on kinematic viscosity, length, and concentration difference of the fluid. On the other hand, there is an inverse relationship between the viscosity and velocity of the fluid. Therefore, the viscosity of the fluid declines once it has increased the magnitude of $G c$ (which as a result causes faster the motion) due to which the velocity profile enhances. Finally, a direct correspondence between factor $G c$ and the velocity profile is shown. The inclination effect on the velocity profile is presented in Fig. 5 . It is observed that $f^{\prime}(\eta)$ diminishes on increasing the inclination. Physically, the strength of the buoyancy force reduces at $\Omega=90$ o which leads the decline in velocity outline. Fig 6 establishes the outcome of Williamson constraint on the velocity outline which displays an converse relation with the velocity contour. The reason behind is reduction of the boundary layer thickness.
Table 2 Values of $-\theta^{\prime}(0)$, $-\phi^{\prime}(0)$ and $C_{f x}(0)$

\begin{tabular}{rrrrrrrrrrrrr}
\hline$N b$ & $N t$ & \multicolumn{1}{c}{$\operatorname{Pr}$} & \multicolumn{1}{c}{$L e$} & $M$ & $N$ & $G r$ & $G c$ & $\gamma_{1}$ & $\Omega$ & $-\theta^{\prime}(0)$ & $-\phi^{\prime}(0)$ & $C_{f x}(0)$ \\
\hline 0.1 & 0.1 & 6.5 & 5.0 & 0.5 & 1.0 & 0.1 & 0.9 & 0.1 & $45^{\circ}$ & 0.9657 & 0.7013 & 0.9881 \\
0.5 & 0.1 & 6.5 & 5.0 & 0.5 & 1.0 & 0.1 & 0.9 & 0.1 & $45^{\circ}$ & 0.1162 & 1.2311 & 1.0424 \\
0.1 & 0.5 & 6.5 & 5.0 & 0.5 & 1.0 & 0.1 & 0.9 & 0.1 & $45^{\circ}$ & 0.4117 & 1.0390 & 0.8155 \\
0.1 & 0.1 & 10.0 & 5.0 & 0.5 & 1.0 & 0.1 & 0.9 & 0.1 & $45^{\circ}$ & 1.0261 & 0.6905 & 0.9844 \\
0.1 & 0.1 & 6.5 & 10.0 & 0.5 & 1.0 & 0.1 & 0.9 & 0.1 & $45^{\circ}$ & 0.8027 & 1.8557 & 1.0795 \\
0.1 & 0.1 & 6.5 & 5.0 & 1.0 & 1.0 & 0.1 & 0.9 & 0.1 & $45^{\circ}$ & 0.9447 & 0.6072 & 1.1825 \\
0.1 & 0.1 & 6.5 & 5.0 & 0.5 & 3.0 & 0.1 & 0.9 & 0.1 & $45^{\circ}$ & 1.1994 & -1.0522 & 0.8842 \\
0.1 & 0.1 & 6.5 & 5.0 & 0.5 & 1.0 & 0.3 & 0.9 & 0.1 & $45^{\circ}$ & 0.9698 & 0.7132 & 0.9445 \\
0.1 & 0.1 & 6.5 & 5.0 & 0.5 & 1.0 & 0.1 & 1.5 & 0.1 & $45^{\circ}$ & 0.9822 & 0.7615 & 0.8229 \\
0.1 & 0.1 & 6.5 & 5.0 & 0.5 & 1.0 & 0.1 & 0.9 & 0.2 & $45^{\circ}$ & 0.9653 & 0.7151 & 1.1418 \\
0.1 & 0.1 & 6.5 & 5.0 & 0.5 & 1.0 & 0.1 & 0.9 & 0.1 & $60^{\circ}$ & 0.9564 & 0.6660 & 1.0715 \\
0.1 & 0.1 & 6.5 & 5.0 & 0.5 & 1.0 & 0.1 & 0.9 & 0.1 & $90^{\circ}$ & 0.9300 & 0.5554 & 1.3015 \\
\hline
\end{tabular}

SN Applied Sciences 


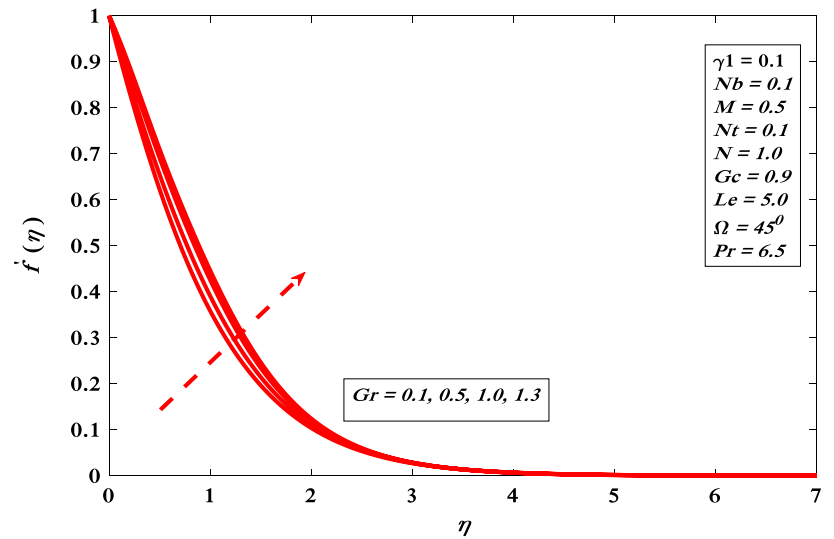

Fig. 3 Velocity $f^{\prime}(\eta)$ verses distinct values of $G r$

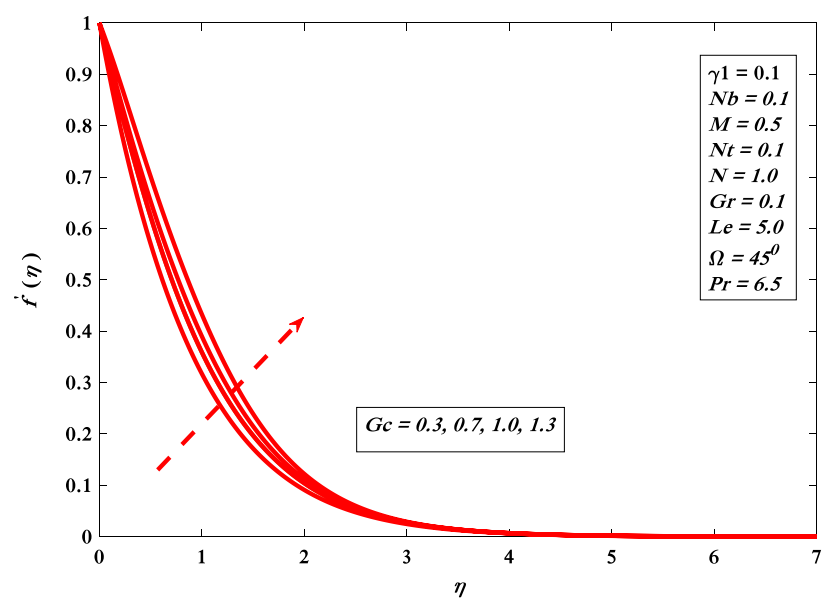

Fig. 4 Velocity $f^{\prime}(\eta)$ verses distinct values of Gc

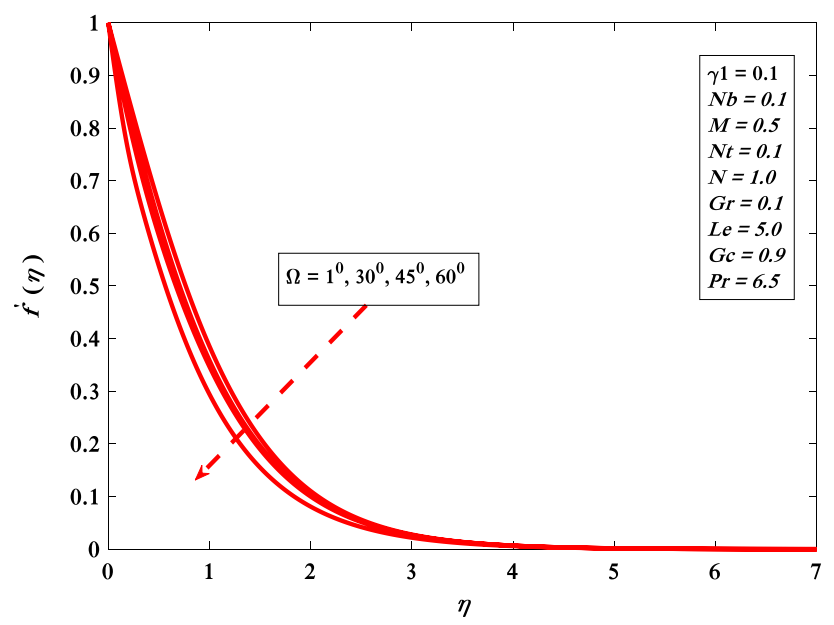

Fig. 5 Velocity $f^{\prime}(\eta)$ verses distinct values of $\Omega$

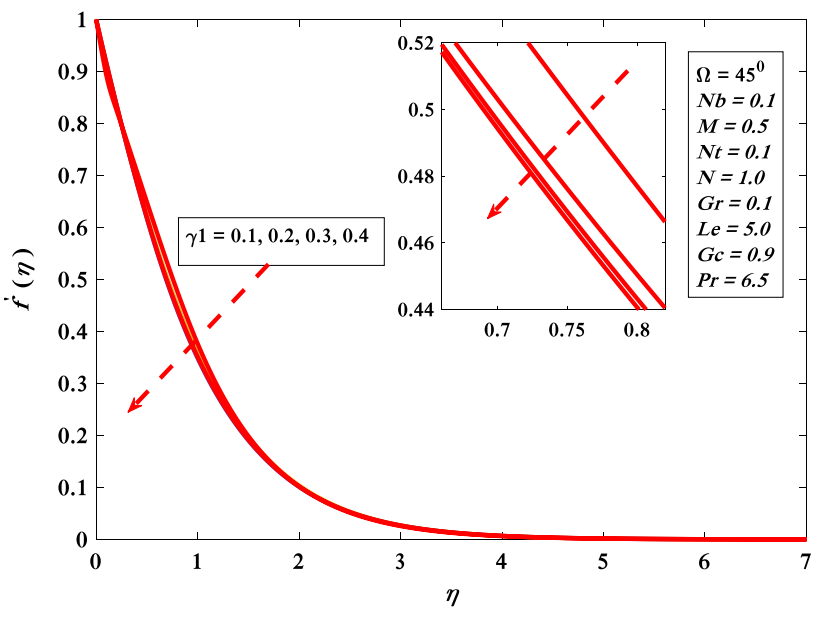

Fig. 6 Velocity $f^{\prime}(\eta)$ verses distinct values of $\gamma_{1}$

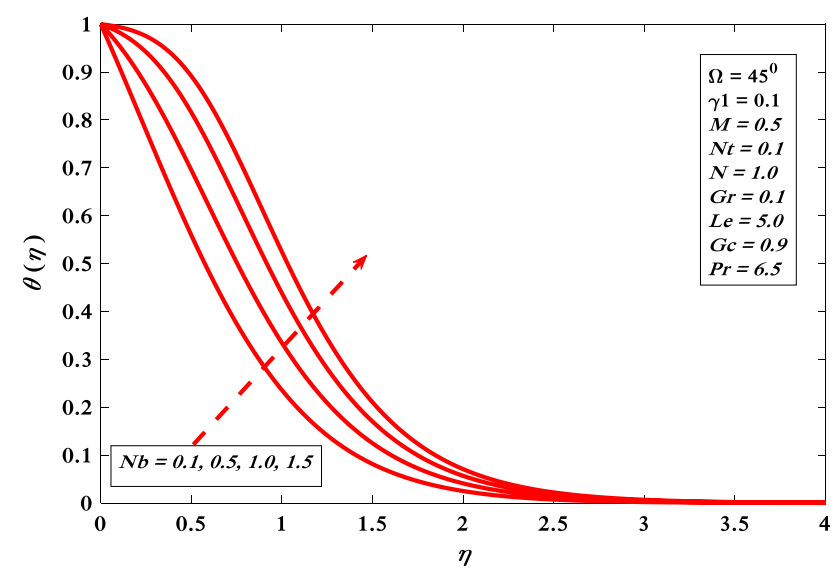

Fig. 7 Temperature $\theta(\eta)$ verses distinct values of $\mathrm{Nb}$

\subsection{Temperature Profile}

Figures 7, 8, 9 and 10 exhibit the temperature profile against incorporated factors. Fig 7 presents $\mathrm{Nb}$ effect on $\theta(\eta)$. The temperature profile shows direct relation with $\mathrm{Nb}$ because Brownian motion warm up the boundary layer which yield the temperature improves. Thermophoretic effect on the temperature profile reveals in Fig. 8. The effect of thermophoresis displays direct correspondence with the temperature profile because the deviation in wall temperature and reference temperature enhanced by enhancing thermophoretic effect. Fig 9 signifies that the temperature profile decreases by growing the factor $\operatorname{Pr}$. This is because the higher magnitudes of $\operatorname{Pr}$ cause the enhancement in viscosity and drop the width of thermal boundary layer. The influence of radiations factor on the temperature is visulaized in Fig. 10. Physically, the conductive energy transport is higher than the radiative energy 


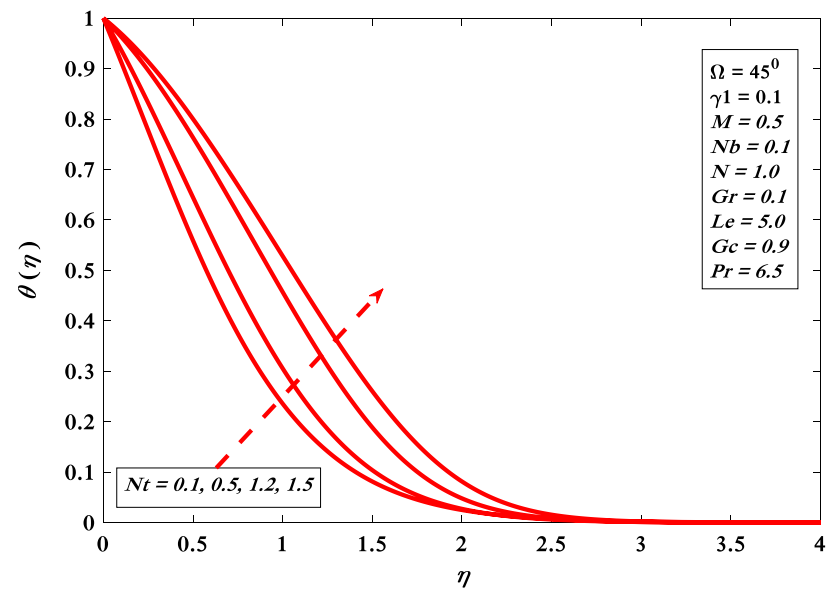

Fig. 8 Temperature $\theta(\eta)$ verses distinct values of $N t$

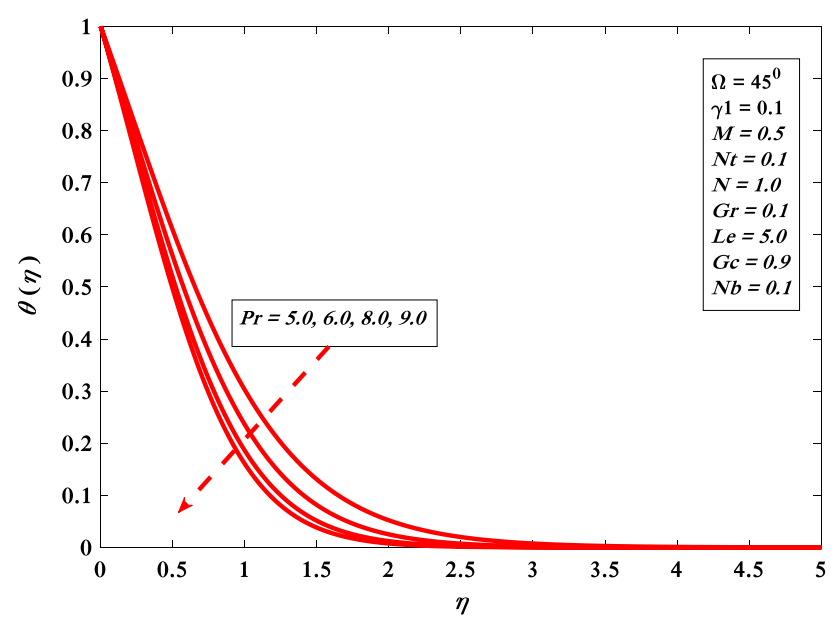

Fig. 9 Temperature $\theta(\eta)$ verses distinct values of $\mathrm{Pr}$

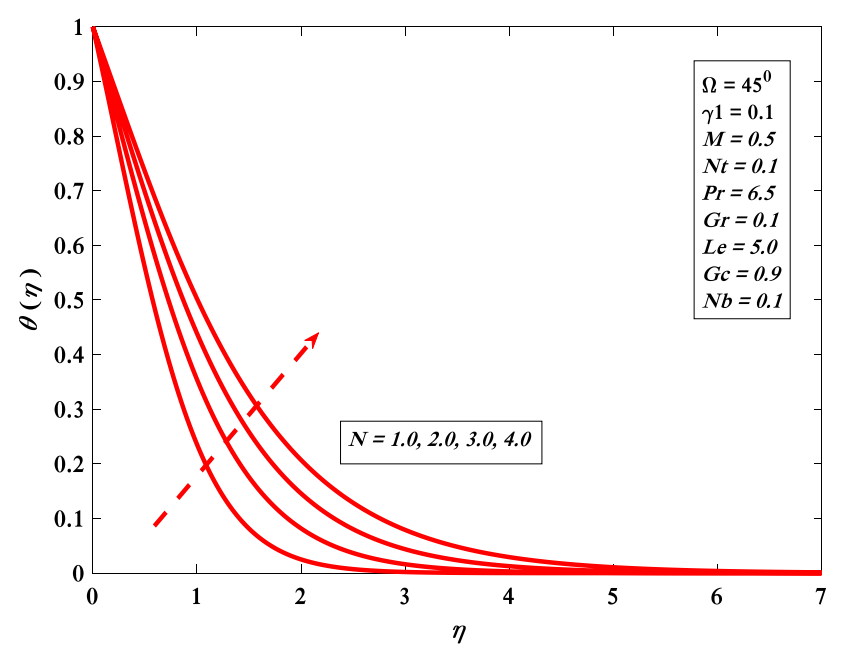

Fig. 10 Temperature $\theta(\eta)$ verses distinct values of $N$

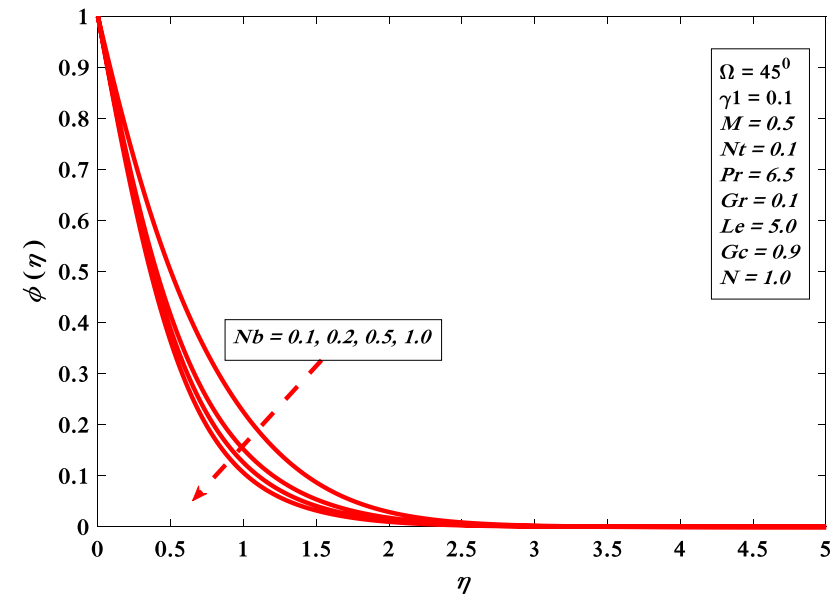

Fig. 11 Concentration $\phi(\eta)$ verses distinct values of $\mathrm{Nb}$

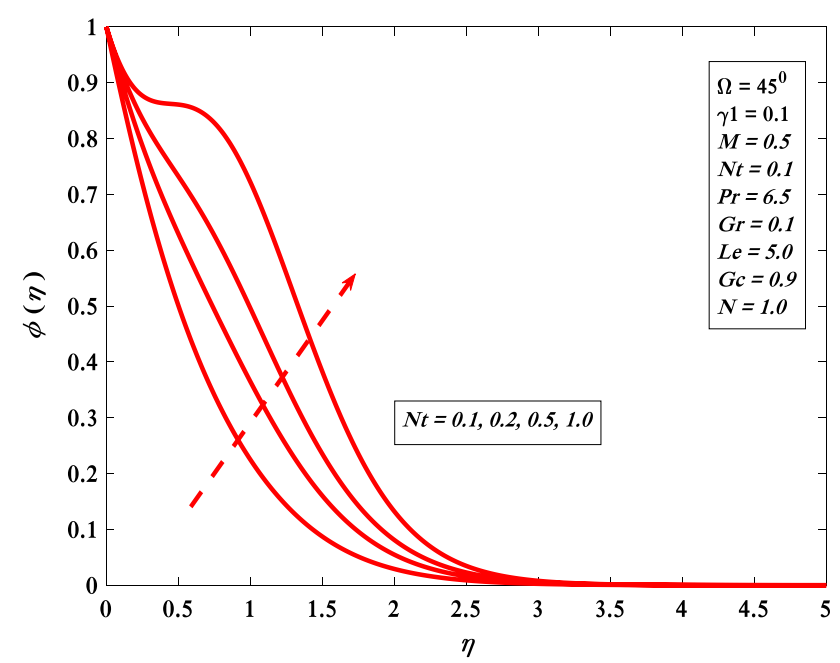

Fig. 12 Concentration $\phi(\eta)$ verses distinct values of $N t$

transport, which causes behind the declining of boundary layer thickness and buoyancy force.

\subsection{Concentration profile}

Figs 11,12 and 13 show the act of concentration profiles for different factors. An improvement in $\mathrm{Nb}$ reduces the boundary layer thickness which lessening the concentration profile (see Fig. 11). Fig 12 specifies the thermophoretic effect on $\phi(\eta)$. It is found from the drawing depicted for the concentration with different magnitudes of $\mathrm{Nt}$ enhanced. Fig 13 show the result of Lewis number on the concentration contour. The boundary layer viscidness moderates by enhancing $L e$. 


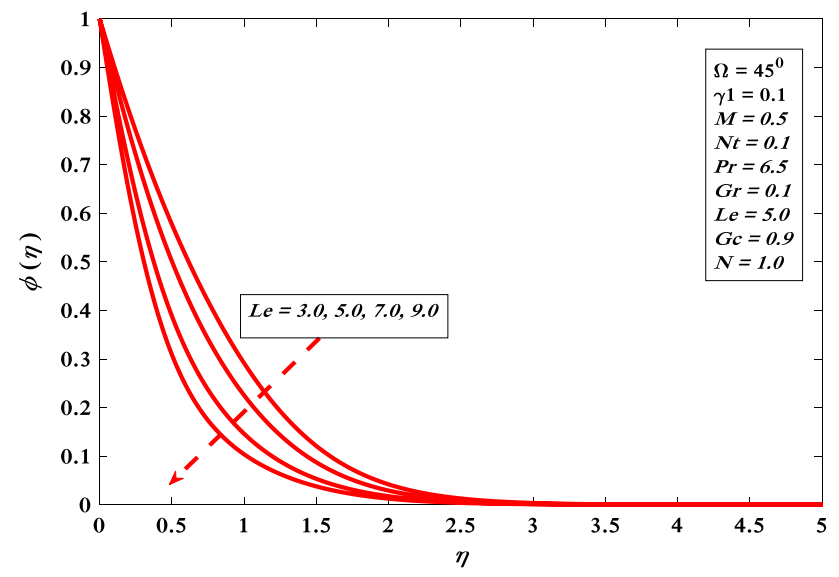

Fig. 13 Concentration $\phi(\eta)$ verses distinct values of $L e$

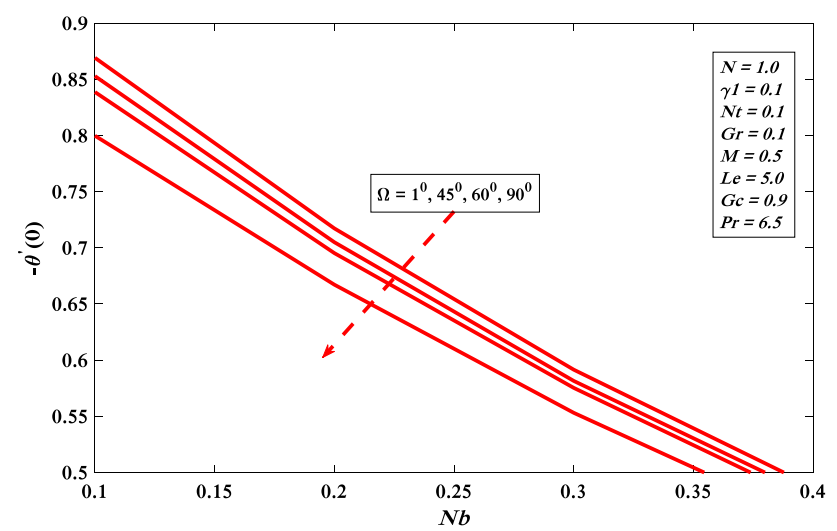

Fig. 14 Heat transport rate $-\theta^{\prime}(0)$ verses distinct values of $\Omega$ and $N b$

\subsection{Heat and Mass Exchange}

Behaviors of heat and mass exchange rates along with skin friction are presented in the Figs. 14, 15, 16, 17, 18 and 19 for altered values of $\Omega, N b$ and $N t$. Figs 14 and 15 demonstrate that the heat and mass exchange rates are diminishes with growth of inclination and Brownian motion impact. On the other hand, the skin friction improves for increasing the Brownian motion and inclination effect portrayed in Fig. 16. Similarly, the heat and mass exchange fluxes are drops against the increasing values of $\mathrm{Nt}$ and $\Omega$ (see Figs. 17, 18). Moreover, wall shear stress enhanced with the growth of $N t$ and $\Omega$ (see Fig. 19).

\section{Conclusions}

In the study under concern, Williamson nanofluid flow generated by the linear stretching inclined surface is investigated. The Brownian motion and thermophoretic

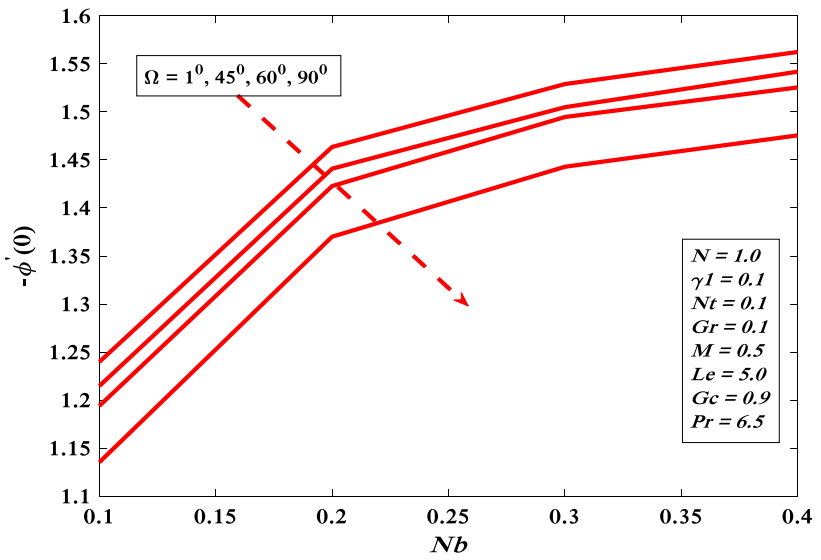

Fig. 15 Mass transport rate $-\phi^{\prime}(0)$ verses distinct values of $\Omega$ and $\mathrm{Nb}$

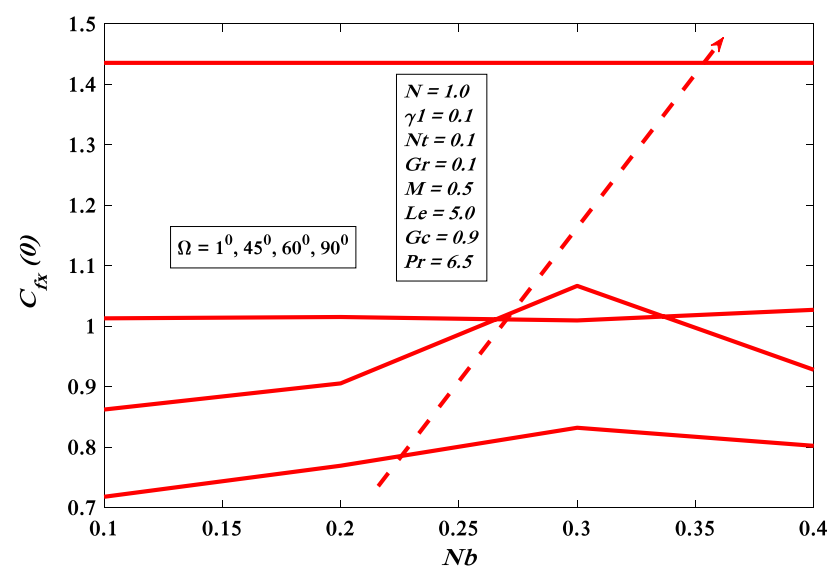

Fig. 16 Skin-friction $C_{f x}(0)$ verses distinct values of $\Omega$ and $N b$

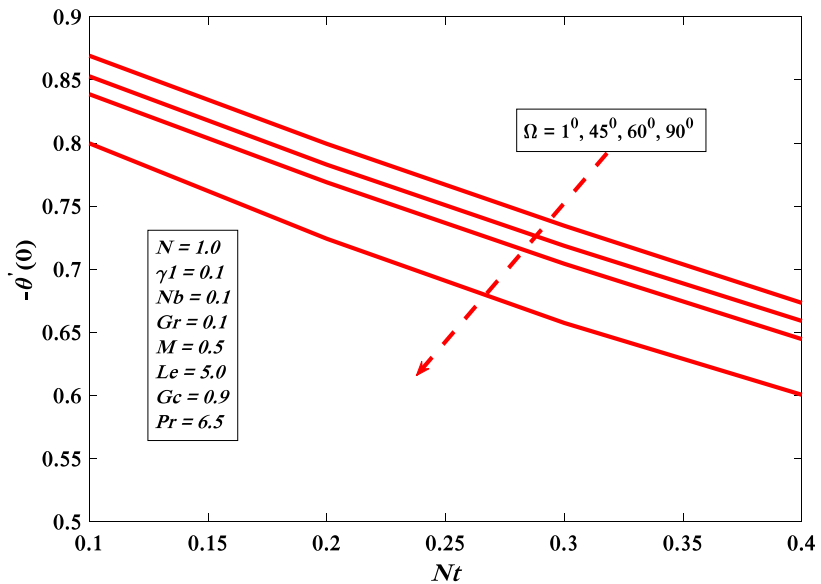

Fig. 17 Heat transport rate $-\theta^{\prime}(0)$ verses distinct values of $\Omega$ and $N t$ 


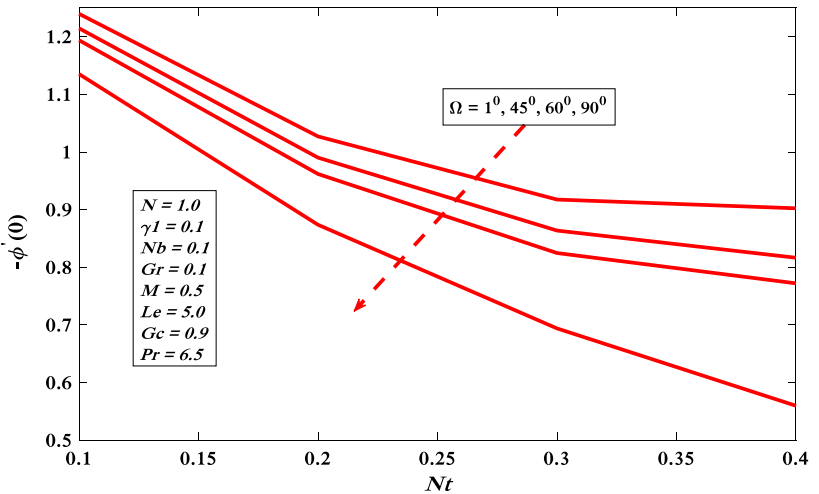

Fig. 18 Mass transport rate $-\phi^{\prime}(0)$ verses distinct values of $\Omega$ and $N t$

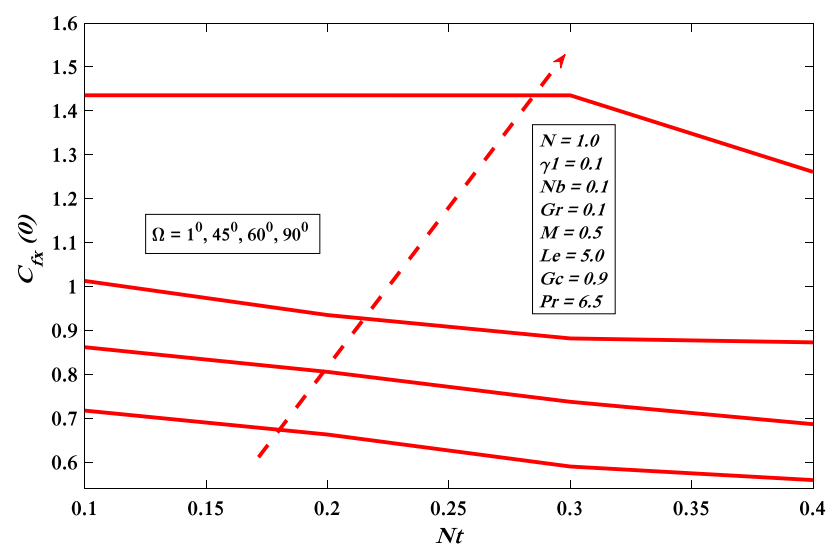

Fig. 19 Skin-friction $C_{f x}(0)$ verses distinct values of $\Omega$ and $N t$

effect are taken into account along with magnetic filed and thermal radiations effect. The boundary layer equations are converted into ordinary nonlinear equations via compatible transformation and utilized the Keller box scheme for the numerical outcomes. The main outcomes of the current study are the following:

- The velocity profile reduces on growing of Williamson factor.

- The growing variations in the thermal radiations enhances the temperature profile.

- Energy and mass transport drop by increasing inclination factor along with Brownian motion parameter.

- The Sherwood number increases with the higher magnitudes of Williamson factor.

- The Nusslet number falls on improving thermophpretic and inclination influence.

- The velocity profile diminishes with the increment in inclination factor.

\section{Compliance with ethical standards}

Conflict of interest The authors declare that they have no conflict of interest.

\section{References}

1. Choi SUS, Eastman JA (1995) Enhancing thermal conductivity of fluids with nanoparticles (No. ANL/MSD/CP-84938; CONF951135-29). Argonne National Lab., Lemont

2. Rafique K, Anwar MI, Misiran M, Khan I, Seikh AH, Sherif ESM, Nisar KS (2019) Brownian motion and thermophoretic diffusion effects on micropolar type nanofluid flow with Soret and Dufour impacts over an inclined sheet: Keller-box simulations. Energies 12:4191

3. Buongiorno J (2006) Convective transport in nanofluids. J Heat Transf 128:240-250

4. Tiwari RK, Das MK (2007) Heat transfer augmentation in a twosided lid-driven differentially heated square cavity utilizing nanofluids. Int J Heat Mass Transf 50:2002-2018

5. Turkyilmazoglu M (2017) Condensation of laminar film over curved vertical walls using single and two-phase nanofluid models. Eur J Mech B Fluids 65:184-191

6. Anwar Ml, Ali M, Rafique K, Shehzad SA (2019) Soret-Dufour and radiative aspects in hydromagnetized nanofluid flow in stratified porous medium. SN Appl Sci 1:1430

7. Chaudhary S, Kanika KM (2019) Impacts of viscous dissipation and Joule heating on hydromagnetic boundary layer flow of nanofluids over a flat surface subjected to Newtonian heating. SN Appl Sci 1:1709

8. Ambreen T, Kim M (2018) Effect of fin shape on the thermal performance of nanofluid-cooled micro pin-fin heat sinks. Int J Heat Mass Transf 126:245-256

9. Liu T, Liu L, Zheng L (2018) Unsteady flow and heat transfer of Maxwell nanofluid in a finite thin film with internal heat generation and thermophoresis. Therm Sci 22:2803-2813

10. Izadi M, Oztop HF, Sheremet MA, Mehryan SAM, Abu-Hamdeh N (2019) Coupled FHD-MHD free convection of a hybrid nanoliquid in an inversed T-shaped enclosure occupied by partitioned porous media. Numer Heat Transf Part A Appl 76:479-498

11. Izadi M, Mohebbi R, Delouei AA, Sajjadi H (2019) Natural convection of a magnetizable hybrid nanofluid inside a porous enclosure subjected to two variable magnetic fields. Int J Mech Sci 151:154-169

12. Shafee A, Haq RU, Sheikholeslami M, Herki JAA, Nguyen TK (2019) An entropy generation analysis for MHD water based Fe3O4 ferrofluid through a porous semi annulus cavity via CVFEM. Int Commun Heat Mass Transf 108:104295

13. Sheikholeslami M (2018) Application of Darcy law for nanofluid flow in a porous cavity under the impact of Lorentz forces. J Mol Liq 266:495-503

14. Turkyilmazoglu M (2019) Fully developed slip flow in a concentric annuli via single and dual phase nanofluids models. Comput Methods Programs Biomed 179:104997

15. Turkyilmazoglu M (2018) Buongiorno model in a nanofluid filled asymmetric channel fulfilling zero net particle flux at the walls. Int J Heat Mass Transf 126:974-979

16. Hsiao K (2016) Stagnation electrical MHD nanofluid mixed convection with slip boundary on a stretching sheet. Appl Therm Eng 98:850-861

17. Hsiao K (2017) Micropolar nanofluid flow with MHD and viscous dissipation effects towards a stretching sheet with multimedia feature. Int J Heat Mass Transf 112:983-990 
18. Turkyilmazoglu M (2017) Mixed convection flow of magnetohydrodynamic micropolar fluid due to a porous heated/ cooled deformable plate: exact solutions. Int J Heat Mass Transf 106:127-134

19. Prasannakumara BC, Gireesha BJ, Krishnamurthy MR, Kumar KG (2017) MHD flow and nonlinear radiative heat transfer of Sisko nanofluid over a nonlinear stretching sheet. Inform Med Unlocked 9:123-132

20. Shehzad SA, Abbas Z, Rauf A (2019) Finite difference approach and successive over relaxation (SOR) method for MHD micropolar fluid with Maxwell-Cattaneo law and porous medium. Phys Scr 94:115228

21. Abbasi FM, Shanakhat I, Shehzad SA (2019) Entropy generation analysis for peristalsis of nanofluid with temperature dependent viscosity and Hall effects. J Magn Magn Mater 474:434-441

22. Pal D, Roy N (2019) Role of Brownian motion and nonlinear thermal radiation on heat transfer of a Casson nanofluid over stretching sheet with slip velocity and non-uniform heat source/ sink. J Nanofluids 8:556-568

23. Ghadikolaei SS, Hosseinzadeh K, Ganji DD, Jafari B (2018) Nonlinear thermal radiation effect on magneto Casson nanofluid flow with Joule heating effect over an inclined porous stretching sheet. Case Stud Therm Eng 12:176-187

24. Saidulu N, Gangaiah T, Lakshmi AV (2019) Radiation effect on MHD flow of a tangent hyperbolic nanofluid over an inclined exponentially stretching sheet. Int J Fluid Mech Res 46:277-293

25. Williamson RV (1929) The flow of pseudoplastic materials. Ind Eng Chem 21:1108-1111

26. Vijayalaxmi T, Shankar B (2016) Hydromagnetic flow and heat transfer of Williamson nanofluid over an inclined exponential stretching sheet in the presence of thermal radiation and chemical reaction with slip conditions. J Nanofluids 5:826-838

27. Khan M, Malik MY, Salahuddin T, Hussian A (2018) Heat and mass transfer of Williamson nanofluid flow yield by an inclined Lorentz force over a nonlinear stretching sheet. Results Phys 8:862-868

28. Kumar KG, Rudraswamy NG, Gireesha BJ, Manjunatha S (2017) Non linear thermal radiation effect on Williamson fluid with particle-liquid suspension past a stretching surface. Results Phys 7:3196-3202

29. Megahed AM (2019) Williamson fluid flow due to a nonlinearly stretching sheet with viscous dissipation and thermal radiation. J Egypt Math Soc 27:12

30. Kumar SG, Varma SVK, Kumar RVMSSK, Raju CSK, Shehzad SA, Bashir MN (2019) Three-dimensional hydromagnetic convective flow of chemically reactive Williamson fluid with non-uniform heat absorption and generation. Int J Chem Reactor Eng 17:20180118

31. Anwar MI, Shafie S, Hayat T, Shehzad SA, Salleh MZ (2017) Numerical study for MHD stagnation-point flow of a micropolar nanofluid towards a stretching sheet. J Braz Soc Mech Sci Eng 39:89-100

32. Khan WA, Pop I (2010) Boundary-layer flow of a nanofluid past a stretching sheet. Int J Heat Mass Transf 53:2477-2483

Publisher's Note Springer Nature remains neutral with regard to jurisdictional claims in published maps and institutional affiliations. 